Mens

Revue d'histoire intellectuelle et culturelle

Frédérick Guillaume Dufour. La sociologie historique :

traditions, trajectoires et débats, Québec, Presses de

l’Université du Québec, 2015, coll. « Politeia », 476 p.

\title{
Patrick-Michel Noël
}

Volume 17, numéro 1-2, automne 2016, printemps 2017

URI : https://id.erudit.org/iderudit/1050800ar

DOI : https://doi.org/10.7202/1050800ar

Aller au sommaire du numéro

Éditeur(s)

Centre de recherche en civilisation canadienne-française

ISSN

1492-8647 (imprimé)

1927-9299 (numérique)

Découvrir la revue

Citer ce compte rendu

Noël, P.-M. (2016). Compte rendu de [Frédérick Guillaume Dufour. La sociologie historique : traditions, trajectoires et débats, Québec, Presses de l'Université du Québec, 2015, coll. « Politeia », 476 p.] Mens, 17(1-2), 207-210.

https://doi.org/10.7202/1050800ar 
Madeleine a des goûts plus classiques; elle apprécie Valéry et Mallarmé, que Marcelle juge comme pratiquant un « art trop cérébral, même décadent " (p. 67). Jacques, quant à lui, prétend que Marcelle ne comprend pas Proust, qu'on gagnerait à connaître par une deuxième lecture. Marcelle, par contre, est davantage emballée par Les cent vingt journées de Sodome de Sade, qui " est à faire déshabiller une sœur " (p. 87), ce que lui reproche d'ailleurs Madeleine, qui associe les lecteurs de Sade à « ceux qui paient 25 \$ à New York pour voir une femme et un chien faire l'amour » (p. 127).

Comme Le droit d'être rebelle présente un choix de lettres et non pas l'entièreté de la correspondance conservée, et qu'il offre un appareil de notes explicatives relativement discret, on lira peut-être surtout ce recueil, en fin de compte, un peu comme s'il s'agissait d'un roman, témoignant certes d'une époque chargée historiquement, mais aussi construisant graduellement les personnages faisant partie de la vaste saga familiale ferronnienne. Le travail d'édition resterait d'ailleurs à être peaufiné pour éliminer les coquilles, rétablir les portions de phrase manquantes et mieux établir le lien entre les lettres lorsque certaines d'entre elles ont été volontairement retirées de la publication. À cet égard, une brève introduction à chacune des sections aurait été souhaitable afin d'offrir une meilleure contextualisation.

- Sophie Marcotte Département d'études françaises Université Concordia

\section{Frédérick Guillaume Dufour. La sociologie historique : traditions, trajectoires et débats, Québec, Presses de l'Uni- versité du Québec, 2015, coll. " Politeia », 476 p.}

Cet ouvrage se veut une introduction au domaine interdisciplinaire de la sociologie historique. Il s'agit, à ce jour, de la seule synthèse disponible en langue française traitant non seulement de l'histoire, de la théorie et de la méthodologie de la sociologie historique, mais aussi des principaux problèmes, objets et thèmes autour desquels 
cette dernière s'est développée sur le plan empirique. On y aborde différentes contributions en provenance des États-Unis, du Canada et de la Grande-Bretagne. L'auteur est professeur au Département de sociologie de l'Université du Québec à Montréal et se spécialise dans l'étude des relations internationales.

Frédérick Guillaume Dufour n'a pas l'ambition de prendre position dans les différents débats structurant le champ de la sociologie historique : il veut plus modestement nous en esquisser un portrait en présentant un survol de ses principales approches et de leur évolution ainsi que de ses principaux enjeux. Il prend bien la peine de souligner que la sociologie historique ne doit pas se penser comme une sous-discipline des sciences sociales, mais plutôt comme "un carrefour de trajectoires disciplinaires et antidiscplinaires en sciences sociales [...] où convergent des chercheurs œuvrant en sociologie (politique et culturelle), en économie institutionnaliste, en politique comparée, en relations internationales, en anthropologie économique et dans différentes branches de l'histoire sociale, démographique, économique, politique et des idées politiques » (p. 3 ; en italique dans le texte). Bien qu'elle ne fasse l'objet d'aucune "définition consensuelle» (p. 3), la sociologie historique se fonde sur certains principes et convictions méthodologiques et théoriques. Sur le plan méthodologique, les tenants de la sociologie historique s'entendent sur la nécessité d'étudier leurs objets dans la longue durée, "soit dans des contextes délimités au moyen d'une périodisation contrôlée par la théorie " (p. 5), en employant une méthode comparative. Sur le plan théorique, ils puisent incontestablement chez les « classiques ", Smith, Marx et Weber, mais aussi chez Barthes, Bourdieu, Corrigan Curtis, Elias, Foucault, Goffman, Gramsci, Sayer, Steinmetz, Tilly. L'auteur soutient d'ailleurs que bon nombre des contributions en sociologie historique cherchent, à partir de références théoriques plus contemporaines, à renouer un dialogue critique avec les classiques.

L'ouvrage se divise en huit chapitres. Le premier se veut une généalogie de la sociologie historique, dans lequel l'auteur discute du lien réciproque entre histoire et sociologie et des différentes 
configurations issues de leur croisement. Le deuxième aborde des enjeux méthodologiques, notamment la méthode comparative et son incidence pour comprendre l'historicité des transformations sociales. Après ces deux chapitres épistémologique et méthodologique - les meilleurs à notre avis -, l'auteur présente en six chapitres quelques objets d'études majeurs de la sociologie historique : questions de classes et de reproduction sociale (chapitre 3), questions de pouvoir et de (trans)formation de l'État (chapitre 4), la transition vers le capitalisme (chapitre 5), les processus révolutionnaires et les conflits sociaux (chapitre 6), les régimes politiques (chapitre 7), nation et nationalisme (chapitre 8).

Cette division et cette organisation du livre permettent aux lecteurs de se retrouver facilement dans le champ de la sociologie historique. Chacun des chapitres présente de façon concise et claire les différents débats et sous-débats des thèmes majeurs de la sociologie historique. En cela l'ouvrage est d'une qualité et d'une pertinence indéniables. Nous pouvons néanmoins adresser trois critiques à l'auteur. Tout d'abord, bien que nous reconnaissions que l'exhaustivité bibliographique soit une illusion, nous nous demandons pourquoi les travaux français en "socio-histoire " n'ont pas été inclus dans les contributions recensées dans l'ouvrage. Par exemple, n'aurait-il pas été pertinent d'intégrer la revue Genèses (et les auteurs qui y sont associés), qui a été fondée explicitement dans le but de programmer une "socio-histoire " ? Ensuite, il nous semble que la notion d'histoire aurait mérité une élucidation épistémologique un peu plus poussée : qu'est-ce que les tenants de la sociologie historique entendent par " histoire ", au-delà de la nécessité (évidente?) de contextualiser les problématiques sociales et de tenir compte de leur évolution? L'épistémologie de l'histoire serait-elle l'angle mort de cette sociologie historique? De même, le projet contemporain de la sociologie historique, présenté à juste titre par l'auteur comme une réaction à «l'antihistoricisme " parsonien (p. 24), aurait-il participé à un processus plus large et englobant que Terrence McDonald et François Dosse ont respectivement nommé le " historic turn in the human sciences" 
et l'" humanisation des sciences humaines ${ }^{1}$ depuis le tournant des années 1970 ?

Enfin, pour autant que l'auteur ait insisté à juste titre sur la réflexivité de la sociologie historique (p. 37 et 86), n’aurait-il pas été pertinent de mettre à l'épreuve ce champ de recherche par les outils et procédés de ce champ? N'aurait-ce pas été là l'ultime défense et illustration de celle-ci? Certes, l'auteur présente une généalogie spontanée du champ, cerne ses fondements théoriques et brosse un riche portrait de sa production empirique qui permettra au lectorat francophone de mieux s'y orienter; cependant, il n'a pas produit une sociologie historique de la sociologie historique. Pourtant, il existe des références théoriques à l'intérieur même de la sociologie historique pour procéder à cette mise en abyme. Pensons à la théorie des champs scientifiques de Bourdieu ou aux science studies anglo-saxonnes. En fait, et c'est là sans doute la principale lacune de l'ouvrage, l'auteur explicite très peu les paramètres méthodologiques de son enquête sur la sociologie historique. Comment a-t-il reconstruit le champ de la sociologie historique? À l'enseigne d'une sociologie, d'une histoire intellectuelle ou d'une histoire philosophante? Pour cette raison, l'ouvrage intéressera plutôt les adeptes et les étudiants de la sociologie historique que ceux qui souhaitent la comprendre comme objet dans le cadre d'une histoire des sciences.

- Patrick-Michel Noël Université de Saint-Boniface

\section{E.-Martin Meunier (dir.). Le Québec et ses mutations cultu- relles : six enjeux pour le devenir d'une société, Ottawa, Les Presses de l'Université d'Ottawa, 2016, 520 p.}

Territoire complexe et diversifié, le Québec d'aujourd'hui est pourtant encore, nous semble-t-il, assez peu connu, du point de vue

${ }^{1}$ Terrence J. McDonald (dir.), The Historic Turn in the Human Sciences, Ann Arbor, The University of Michigan Press, 1996; François Dosse, L'empire du sens : l'humanisation des sciences humaines, Paris, La Découverte, 1997. 